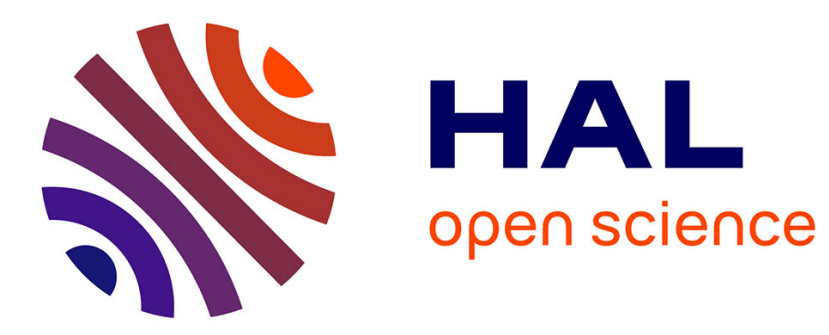

\title{
Storm-induced energization of radiation belt electrons: Effect of wave obliquity
}

A.V. Artemyev, O. V. Agapitov, D Mourenas, V Krasnoselskikh, L. M. Zelenyi

\section{To cite this version:}

A.V. Artemyev, O. V. Agapitov, D Mourenas, V Krasnoselskikh, L. M. Zelenyi. Storm-induced energization of radiation belt electrons: Effect of wave obliquity. Geophysical Research Letters, 2013, 40, pp.4138-4143. 10.1002/grl.50837 . insu-01178863

\section{HAL Id: insu-01178863 \\ https://hal-insu.archives-ouvertes.fr/insu-01178863}

Submitted on 21 Jul 2015

HAL is a multi-disciplinary open access archive for the deposit and dissemination of scientific research documents, whether they are published or not. The documents may come from teaching and research institutions in France or abroad, or from public or private research centers.
L'archive ouverte pluridisciplinaire HAL, est destinée au dépôt et à la diffusion de documents scientifiques de niveau recherche, publiés ou non, émanant des établissements d'enseignement et de recherche français ou étrangers, des laboratoires publics ou privés. 


\title{
Storm-induced energization of radiation belt electrons: Effect of wave obliquity
}

\author{
A. V. Artemyev, ${ }^{1,2}$ O. V. Agapitov, ${ }^{1,3}$ D. Mourenas, ${ }^{4}$ \\ V. Krasnoselskikh, ${ }^{1}$ and L. M. Zelenyi ${ }^{2}$ \\ Received 22 July 2013; revised 06 August 2013; accepted 07 August 2013; published 20 August 2013.
}

[1] New Cluster statistics allow us to determine for the first time the variations of both the obliquity and intensity of lower-band chorus waves as functions of latitude and geomagnetic activity near $L \sim 5$. The portion of wave power in very oblique waves decreases during highly disturbed periods, consistent with increased Landau damping by inward-penetrating suprathermal electrons. Simple analytical considerations as well as full numerical calculations of quasi-linear diffusion rates demonstrate that early-time electron acceleration occurs in a regime of loss-limited energization. In this regime, the average wave obliquity plays a critical role in mitigating lifetime reduction as wave intensity increases with geomagnetic activity, suggesting that much larger energization levels should be reached during the early recovery phase of storms than during quiet time or moderate disturbances, the latter corresponding to stronger losses. These new effects should be included in realistic radiation belt simulations. Citation: Artemyev, A. V., O. V. Agapitov, D. Mourenas, V. Krasnoselskikh, and L. M. Zelenyi (2013), Storm-induced energization of radiation belt electrons: Effect of wave obliquity, Geophys. Res. Lett., 40, 4138-4143, doi:10.1002/grl.50837.

\section{Introduction}

[2] The rapid energization of trapped electrons up to $\mathrm{MeVs}$ in Earth's radiation belts is a puzzling problem of plasma physics. Accurately modeling their dynamics is an important task as such "killer" electrons represent a known hazard to satellite assets [Horne et al., 2013]. Although much progress has been achieved in the preceding decades in understanding and evaluating the processes of radial diffusion, convection, magnetopause shadowing at large $L$-shells, and especially quasi-linear pitch angle and energy diffusion of particles by intense whistler-mode waves [e.g., Shprits et al., 2008b], important discrepancies still remain between Fokker-Planck code results and satellite observations of electrons flux variations [e.g., Horne et al., 2013]. While basic physical phenomena are better known today, the critically needed accurate and comprehensive data about wave and plasma parameters are only progressively

\footnotetext{
Additional supporting information may be found in the online version

${ }^{1}$ LPC2E/CNRS, University of Orleans, Orleans, France.

${ }^{2}$ Space Research Institute, RAS, Moscow, Russia.

${ }^{3}$ National Taras Shevchenko, University of Kiev, Kiev, Ukraine.

${ }^{4}$ CEA, DAM, DIF, Arpajon, France.
} of this article.

Corresponding author: A. V. Artemyev, Space Research Institute, RAS, Moscow 117997, Russia. (ante0226@gmail.com)

(C)2013. American Geophysical Union. All Rights Reserved. 0094-8276/13/10.1002/grl.50837 becoming available thanks to more and more sophisticated spacecrafts such as Cluster or the Van Allen probes [Agapitov et al., 2013; Baker et al., 2013].

[3] Roughly speaking, electron energization is produced by the combined effects of adiabatic heating in the course of radial transport and wave-particle resonant interactions. However, the presence of a peak of high-energy electron flux around $L \sim 5.5$ suggests the dominance of waveparticle interactions in this process [Chen et al., 2007]. The most favorable period for producing a high-energy electron population is a geomagnetic storm, during which whistlermode chorus wave intensity increases by three to four orders of magnitude [Horne et al., 2005; Shprits et al., 2007]. However, wave growth enhances electron scattering and induced losses as well (see discussion in Shprits et al. [2008b]). The competition between energization and losses can slow down or reduce electron acceleration. In this paper, we show that this problem is made even more complex in the real magnetosphere by the increase of the dimensions of the parameter space of the system. In the outer radiation belt, it is indeed necessary to take into account not only the variation of the lower-band chorus wave intensity with $D_{s t}$ but also the evolution of the wave-normal angle distribution in the course of a storm. Oblique waves substantially influence particle lifetimes and determine their energization timescales. As concerns lifetimes, the increase of the wave amplitude can be almost compensated by the decrease of the oblique wave population during a storm, an effect which has not been taken into account in previous studies.

\section{Spacecraft Statistics}

[4] We make use of 10 years of Cluster observations to determine the distribution of lower-band chorus wavenormal angles and root-mean-square (RMS) wave amplitudes on the dayside at $L \sim 5$ for magnetic latitudes $\lambda<45^{\circ}$ and different $D_{s t}$ ranges. The STAFF-SA instrument onboard Cluster allows to obtain the angle $\theta$ between the direction of wave propagation and the background magnetic field (see the statistical study in Agapitov et al. [2012] and Agapitov et al. [2013]). We consider three $D_{s t}$ ranges: $\left|D_{s t}\right|<10, D_{s t} \in$ $[-40,-10]$, and $D_{s t} \in[-80,-40] \mathrm{nT}$. The corresponding distributions of wave amplitudes and wave-normal angles in 2$\mathrm{D}$ space $(\lambda, \theta)$ are displayed in Figure 1. The $\theta$ range can be split into two parts corresponding to oblique $\left(\theta=60^{\circ}-90^{\circ}\right)$ and nearly parallel $\left(\theta<45^{\circ}\right)$ waves. This separation corresponds to different roles played by these wave populations in particle scattering and acceleration [Mourenas et al., 2012a, 2012b].

[5] For quiet conditions $\left|D_{s t}\right|<10 \mathrm{nT}$, there are no oblique waves in the vicinity of the equator, but both the 


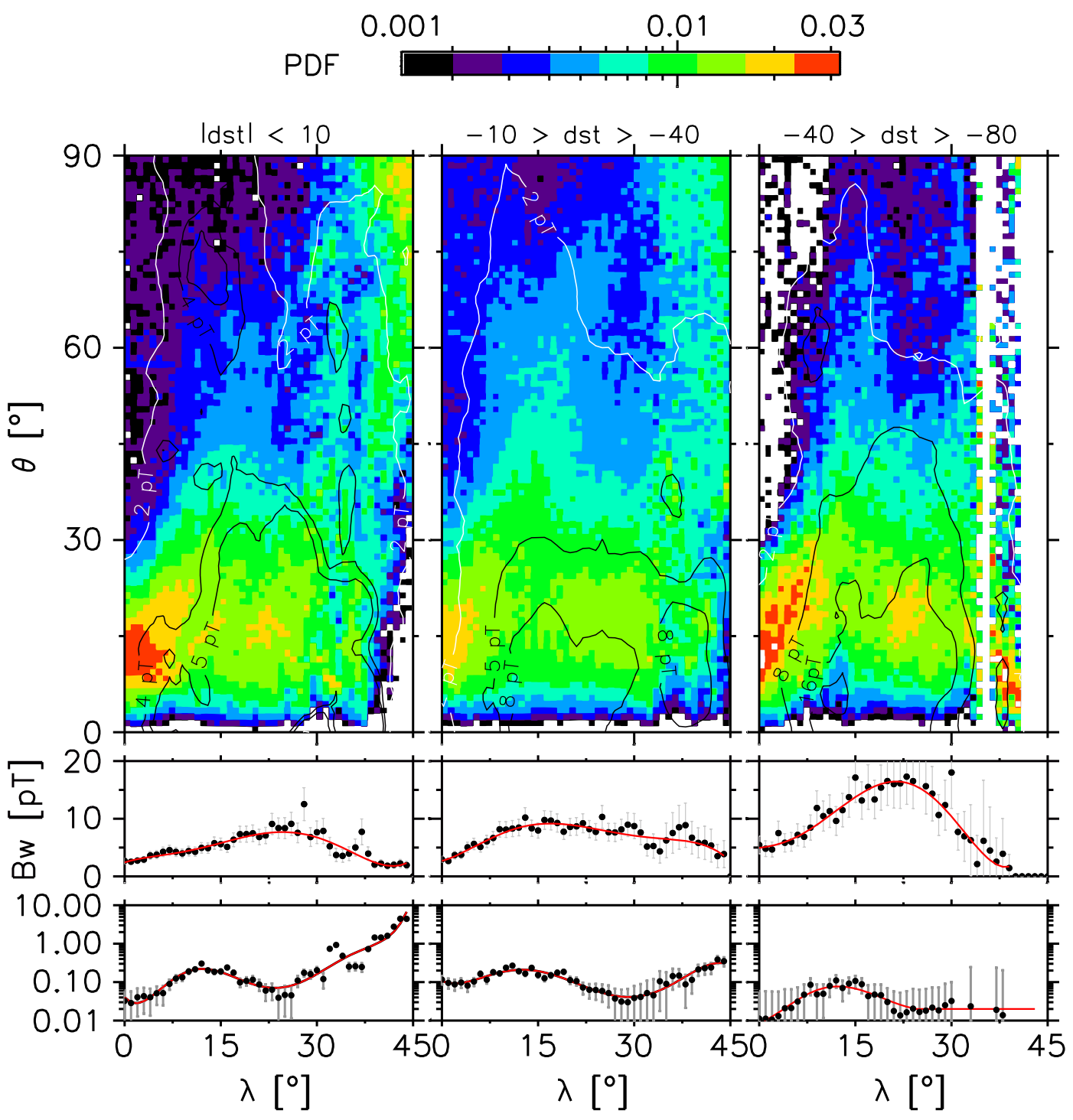

Figure 1. Distributions of RMS wave amplitudes and wave-normal angles for three $D_{s t}$ ranges. (top) The probability density function of wave occurrences as well as amplitudes in the $(\lambda, \theta)$ domain. (middle) The full (three components) wave amplitude as a function of latitude: spacecraft data (black circles) and approximations (red curves). (bottom) The oblique $\left(\theta>60^{\circ}\right)$ to parallel $\left(\theta<45^{\circ}\right)$ wave amplitude ratio (using wave intensities weighted by occurrences) as a function of latitude: spacecraft data (black circles) and approximations (red curves).

occurrences and average intensity of oblique waves, and the oblique to parallel wave amplitude ratio, rapidly increase with $\lambda$ (in agreement with ray-tracing results [see Chen et al., 2013a]). At $\lambda>30^{\circ}$, most of the waves are very oblique. Such a $\theta$ distribution results in a significant intensification of electron pitch angle scattering as it involves higher-order cyclotron resonances [Artemyev et al., 2012]. The range $-40<D_{s t}(\mathrm{nT})<-10$ corresponds to a larger power fraction $(\sim 2 \%)$ of oblique waves near the equator; however, their amplitudes are now substantially smaller at high $\lambda$, certainly due to increased Landau damping [Chen et al., 2013a]. The decrease of the oblique wave population is stronger than the general increase of wave intensity with $\left|D_{s t}\right|$, and thus, the impact of oblique waves on particle scattering slightly decreases with $\left|D_{s t}\right|$. In the highest $\left|D_{s t}\right|$ range $\left(-80<D_{s t}(\mathrm{nT})<-40\right)$, oblique waves are only present at intermediate latitudes - their amplitudes are less than $10 \%$ of the parallel wave amplitude at $\lambda \sim 20^{\circ}$, dropping at higher latitudes. The decrease of oblique wave intensity at high latitudes (where oblique waves are most effective [see Artemyev et al., 2013]) should result in a decrease of pitch angle scattering rates, partly compensating the increase of parallel wave intensity.

[6] In this paper we use the data presented in Figure 1 to calculate pitch angle and energy diffusion coefficients. The $\theta$ distribution is approximated by two Gaussians with mean values and variances depending on $\lambda: g(\theta, \lambda)=g_{1}(\theta, \lambda)+$ $10^{2 A(\lambda)} g_{2}(\theta, \lambda)$, where $g_{i}=\exp \left(-\left(\theta-\theta_{m i}(\lambda)\right)^{2} / \theta_{w i}^{2}(\lambda)\right)$ and $i=1,2$. The coefficient $A(\lambda)$ is obtained from mean-squareroot fits to the ratios shown in the bottom panels of Figure 1 (see supporting information). Functions $\theta_{m i}(\lambda), \theta_{w i}(\lambda)$ vary with $\lambda$ and $D_{s t}$ only weakly: $\theta_{m 1}(\lambda) \approx 15^{\circ}, \theta_{m 2}(\lambda) \approx 75^{\circ}$, $\theta_{w 1}(\lambda) \approx \theta_{w 2}(\lambda) \approx 10^{\circ}$. For the calculation of diffusion coefficients, integration over $\theta$ is limited to $\tan \theta<$ $0.999 \tan \theta_{r}$, where the resonance cone angle $\theta_{r}$ is calculated from the Appleton-Hartree whistler-mode dispersion 
[Artemyev et al., 2013]. Although warm plasma kinetic effects [Chen et al., 2013b] are not taken into account, our $\theta$ integration is also restricted to refractive index values smaller than 300 .

[7] The total wave amplitude as a function of $\lambda$ is obtained by approximating the data displayed in the middle panel of Figure 1 (see supporting information). We assume that wave spectra are the same for oblique and parallel waves and take them from Horne et al. [2005] - the peak frequency $\omega_{m}$ and variance are 0.35 and 0.15 of the equatorial electron gyrofrequency $\Omega_{c e 0}$. For simplicity, the variation of $\omega_{m}$ with latitude [Bunch et al., 2013] is not taken into account, as lifetimes and acceleration vary weakly with it (see section 3 ). Similarly, plasma frequency $\Omega_{p e}$ is taken as a constant along field lines. Using a more realistic density variation at high latitudes [Denton et al., 2006] has been checked to change lifetimes and acceleration rates by less than $30 \%$.

[8] A dayside plasmapause location at $L \leq 4.5$ for $K_{p}>1$ (corresponding to $D_{s t}<-2 \mathrm{nT}$ ) has been assumed, consistent with mean Combined Release and Radiation Effects Satellite values on the dayside shown in Figure 2 in the work by O'Brien and Moldwin [2003]. Thus, the considered region at $L \sim 5$ lies outside of the compressed plasmasphere. The average equatorial plasma density in the trough can be taken as $N_{e} \sim 100(3 / L)^{4} \mathrm{~cm}^{-3}$ with negligible change from low to high geomagnetic activity [Sheeley et al., 2001].

\section{Electron Lifetimes and Energization as a Function of Parameters}

[9] Electron lifetimes can be obtained by integration of the inverse of the azimuthal drift-averaged pitch angle diffusion rate [Albert and Shprits, 2009]. Recent Cluster statistics [Agapitov et al., 2013] show that lower-band chorus waves are confined to lower latitudes on the nightside than on the dayside (due to larger Landau damping [see Li et al., 2011]) but with similar amplitudes. It implies that nightside diffusion is similar to (or smaller than) dayside diffusion. For the sake of simplicity, lifetime can therefore be estimated as roughly equal (within a factor of 2) to the lifetime calculated for dayside wave parameters. When very oblique waves $\left(\theta>60^{\circ}\right)$ are present only at $\lambda<20^{\circ}$, analytical considerations as well as numerical simulations show that lifetimes can be estimated as $\approx 1 / 3-2 / 3$ of parallel wave lifetimes [Artemyev et al., 2013], yielding

$$
\tau_{L}[\mathrm{~s}] \approx \frac{110\left[\mathrm{pT}^{2} \cdot \mathrm{s}^{2} / \mathrm{rad}\right]}{B_{w}^{2}} \frac{p^{14 / 9} \gamma \omega_{m}^{7 / 9} \Omega_{p e}^{14 / 9}}{\Omega_{c e 0}^{12 / 9}}
$$

at $L \sim 5$ for $\left|D_{s t}\right|>40$, where from now on bounce-averaged RMS wave amplitude $B_{w}$ is in pT, angular frequencies are in $\mathrm{rad} / \mathrm{s}, \gamma$ is the relativistic factor, and $p=\left(\gamma^{2}-1\right)^{1 / 2}$. Lifetimes in equation (1) vary roughly like $\left(E N_{e}\right)^{7 / 9}$ for $E<0.5 \mathrm{MeV}$.

[10] When very oblique chorus waves $\left(\theta>60^{\circ}\right)$ are present up to high latitudes as in the day sector at $L \sim 5$ for $\left|D_{s t}\right|<40$, an analytical estimate of $\tau_{L}$ is [Mourenas et al., 2012a; Artemyev et al., 2013]

$$
\tau_{L}[\mathrm{~s}] \approx \frac{35\left[\mathrm{pT}^{2} \cdot \mathrm{s}^{2} / \mathrm{rad}\right] \gamma p \Omega_{p e}}{B_{w}^{2}} .
$$

In the latter case, one has $\tau_{L} \propto \sqrt{E N_{e}}$ for $E<0.5 \mathrm{MeV}$.

[11] Electron acceleration is important for equatorial pitch angle $\alpha_{0}>50^{\circ}$ because (1) such electrons remain trapped as their pitch angle is preferentially increased via cyclotron diffusion and (2) high pitch angle electrons are generally more abundant [e.g., see Mourenas et al., 2012b, and references therein]. The quasi-linear energy diffusion rate of parallel chorus waves at low latitudes reads as

$$
\left\langle\frac{D_{E E}}{E^{2}}\right\rangle_{B}\left[\frac{1}{\mathrm{~s}}\right] \approx \frac{B_{w}^{2} \Omega_{c e 0}^{3 / 2} \omega_{m}^{1 / 2} \Omega_{p e}^{-3}(\gamma+1)^{1 / 2} \sin \alpha_{0}}{100\left[\mathrm{pT}^{2} \cdot \mathrm{s}^{2} / \mathrm{rad}\right] \tan \Delta \theta \gamma(\gamma-1)^{3 / 2}}
$$

with $\Delta \theta \sim 30^{\circ}$ the wave-normal angle spread [Mourenas et al., 2012b]. We henceforth take analytical $\left\langle D_{E E}\right\rangle_{B}=$ $\left\langle D_{E E}\right\rangle_{B}\left(\alpha_{0} \sim 65^{\circ}-80^{\circ}\right)$ where $B_{w}^{2}$ in (3) is the average wave intensity at latitudes $\lambda<12^{\circ}$ corresponding to high $\alpha_{0}$ cyclotron resonance. Based on Cluster chorus statistics at such low latitudes [Agapitov et al., 2013], energy diffusion rates should be roughly similar in the day and night sectors.

[12] Since $\left\langle D_{E E}\right\rangle_{B} \propto \sqrt{E}$ for $E<0.5 \mathrm{MeV}$ changes very much like $\tau_{L}$, it is reasonable to assume to first order that they are both weakly varying with $E$. We further assume that the evolution of the trapped electron distribution function $F$ can be described by a Fokker-Planck diffusion equation with quasi-linear bounce-averaged isotropic energy and pitch angle diffusion coefficients [Horne et al., 2005]:

$$
\frac{\partial F}{\partial t}=\frac{\partial}{\partial E}\left(A(E)\left\langle D_{E E}\right\rangle \frac{\partial}{\partial E} \frac{F}{A(E)}\right)-\frac{F}{\tau_{L}}
$$

with $A(E)=\left(E+m_{e} c^{2}\right) \sqrt{\left(E+2 m_{e} c^{2}\right) E}$. Although mixed diffusion can be important for particle energization [e.g., Albert, 2009], we omit this effect to consider solutions proposed by Balikhin et al. [2012] for an initially cold distribution without high-energy electrons. The approximation of an initially cold electron distribution is supported by observations of high-energy electron evacuation from the outer radiation belt region at the beginning of storms [Turner et al., 2013; Baker et al., 2013]. Such an assumption allows to obtain simplified analytical solutions without taking into account the dependence of energy diffusion on the gradients in the energy spectrum. Contrary to Balikhin et al. [2012] which assumed infinite lifetimes (i.e., no losses), finite lifetime effects must however be taken into account here. Thus, the early-time broadening of the electron distribution can take a simple form

$$
F(t) \propto E^{-4(1-\beta)} t^{-\beta} \exp \left(-\frac{E^{2}}{4\left\langle D_{E E}\right\rangle_{B} t}-\frac{t}{\tau_{L}}\right),
$$

with $\beta \sim 5 / 4$ for $E<0.5 \mathrm{MeV}$ and $\beta \sim 3 / 2$ for $E>1 \mathrm{MeV}$. A careful inspection of (5) shows that the early-time electron energization is determined by the term $\tau_{L}\left\langle D_{E E}\right\rangle_{B} / E^{2}$. For $\tau_{L}\left\langle D_{E E}\right\rangle_{B} / E^{2} \gg 1$, losses due to pitch angle scattering have no significant influence and early-time electron acceleration at a given energy increases with $\left\langle D_{E E}\right\rangle_{B}$, independently of $\tau_{L}$ (regime of negligible losses). Conversely, for $\tau_{L}\left\langle D_{E E}\right\rangle_{B} / E^{2} \ll 1$, electron losses to the ionosphere should strongly curtail the maximum available energization (loss-dominated regime). In this regime, the maximum energization at a given $E$ now increases with $\tau_{L}\left\langle D_{E E}\right\rangle_{B} / E^{2}$, the peak value of $F$ in equation (5) varying roughly like $\exp \left(-1 / \sqrt{\tau_{L}\left\langle D_{E E}\right\rangle_{B} / E^{2}}\right)$.

\section{Numerical Calculations and Interpretation}

[13] We use wave-normal angle distributions $g(\theta, \lambda)$ approximated by a sum of two Gaussians to calculate 

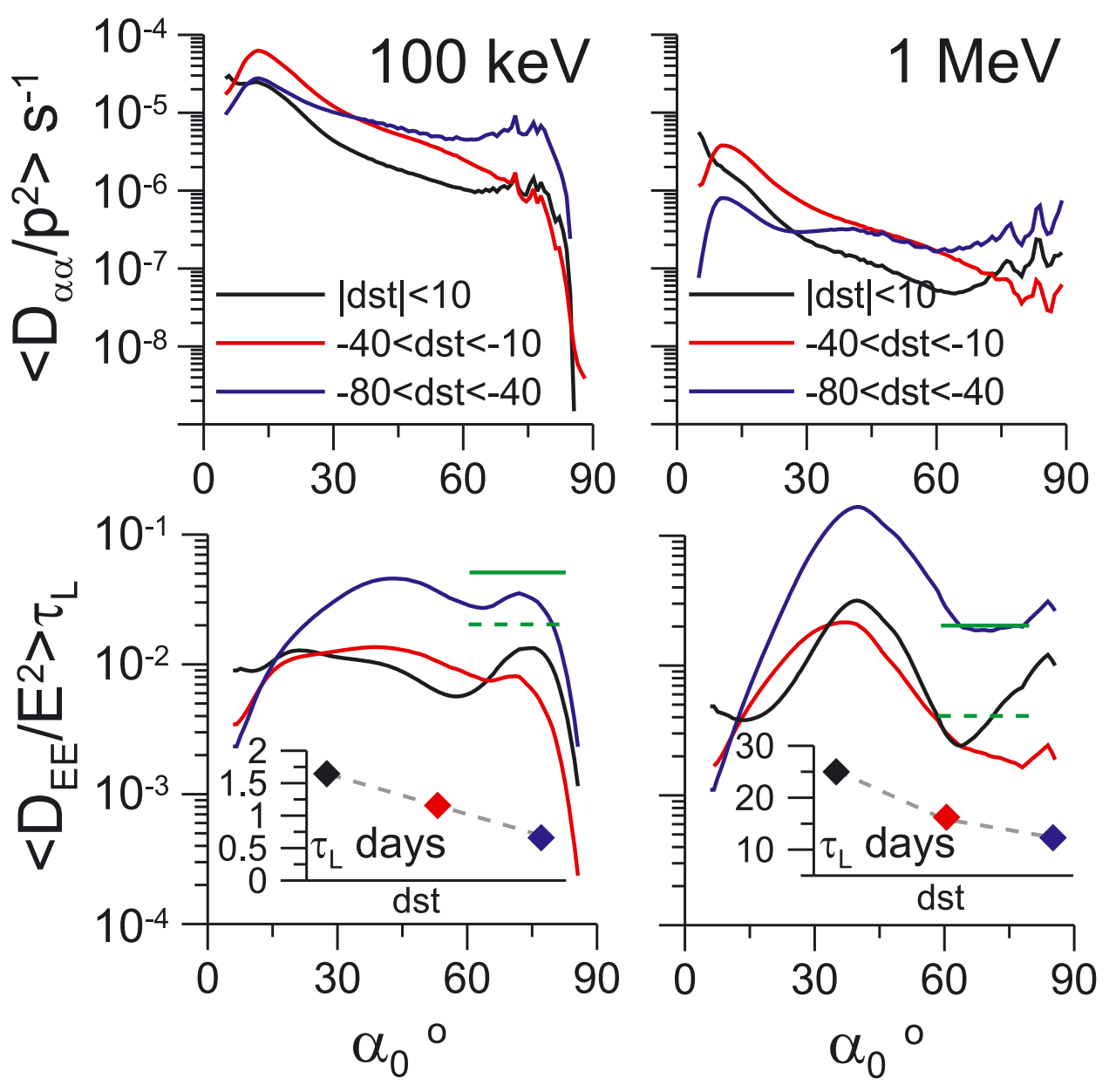

Figure 2. (top) Pitch angle diffusion coefficients for three $D_{s t}$ ranges and two energies. (bottom) $\left\langle D_{E E} / E^{2}\right\rangle \tau_{L}$ as a function of $\alpha_{0}$ for three $D_{s t}$ ranges and two energies. Green lines show analytical estimates (2)-(3) for $\left|D_{s t}\right|<10 \mathrm{nT}$ (dashed line) and (1)-(3) for $D_{s t} \in[-80,-40] \mathrm{nT}$ (solid line). Inserted panels show lifetimes $\tau_{L}$ in the three corresponding $D_{s t}$ ranges (colors are the same as in the main figure).

diffusion coefficients according to a numerical scheme given by Horne et al. [2005] and Mourenas et al. [2012a], considering the Appleton-Hartree whistler-mode dispersion [Artemyev et al., 2013]. The numerical bounceaveraged pitch angle diffusion rate $\left\langle D_{\alpha \alpha}\right\rangle_{B}$, lifetime $\tau_{L}$, and $\tau_{L}\left\langle D_{E E}\right\rangle_{B} / E^{2}$ are plotted in Figure 2 for $E=0.1$ and 1 $\mathrm{MeV}$ in the three considered $D_{s t}$ ranges at $L \sim 5$, using the fits to Cluster wave data detailed above. The energy diffusion rates show broad maxima from $\alpha_{0} \sim 15^{\circ}$ to $80^{\circ}$. The lifetime is obtained from the numerical $\alpha_{0}$ integration of $1 /\left(4\left\langle D_{\alpha \alpha}\right\rangle_{B} \tan \alpha\right)$ from the loss-cone angle up to $\sim 83^{\circ}$ [Albert and Shprits, 2009; Mourenas et al., 2012a; Artemyev et al., 2013], considering that other kinds of observed waves, such as upper-band chorus, fast magnetosonic, or lower frequency whistlers, although less intense, should be sufficient to partly fill the deep gap in pitch angle diffusion near $90^{\circ}$ [e.g., see $\mathrm{Ni}$ et al., 2011; Meredith et al., 2012]. Analytical estimates of $\tau_{L}\left\langle D_{E E}\right\rangle_{B} / E^{2}$ at $\alpha_{0}>60^{\circ}$ from equations (1)-(3) are also plotted, showing a reasonable agreement with simulations. Remarkably, all the cases studied here on the basis of full Cluster statistics [Agapitov et al., 2013] are seen to correspond to the regime $\tau_{L}\left\langle D_{E E}\right\rangle_{B} / E^{2} \ll 1$ of loss-limited electron energization. This contrasts with some important studies which found acceleration rates to exceed loss rates as a consequence of their assumption of parallel chorus [Horne et al., 2005].

[14] It is worth emphasizing that $\tau_{L}\left\langle D_{E E}\right\rangle_{B} / E^{2}$ is independent of wave power for a fixed low latitude to full bounce-average wave intensity ratio. It should therefore remain roughly constant as a function of $D_{s t}$ for nearly constant wave obliquity, such that $\theta \leq 45^{\circ}$ at all geomagnetic activity levels. The very important variation of $\tau_{L}\left\langle D_{E E}\right\rangle_{B} / E^{2}$ with $D_{s t}$ revealed in Figure 2 actually stems from the reduction of the obliqueness of lower-band chorus waves during the most active periods considered here.

[15] Firstly, $\tau_{L}\left\langle D_{E E}\right\rangle_{B} / E^{2}$ is slightly diminished at $\alpha_{0}>50^{\circ}$ as $D_{s t}$ decreases from the range $[-10,10]$ to the range $[-40,-10] \mathrm{nT}$. In the latter $D_{s t}$ range, a significant part of chorus wave power resides in oblique waves at latitudes above $10^{\circ}$. Lifetimes drop roughly $30 \%$ due to rising wave power from quiet time to substorm-range at moderate to high latitudes. Meanwhile, energy diffusion is less increased at high $\alpha_{0}$ corresponding to low latitude $\left(<10^{\circ}\right)$ resonance, because the augmentation of the intensity of quasi-parallel waves derived from Cluster statistics is much less important near the equator than at higher latitudes. Thus, electron 
losses are expected to increase in the moderate geomagnetic activity range, while energization should remain roughly the same as during quiet times.

[16] Next, we consider the $D_{s t}$ range from -80 to $-40 \mathrm{nT}$. While this range (as well as the others) is not uniquely related to a specific phase of storms, it should statistically correspond mainly to the early recovery phase of moderate to large storms, although it includes also the shorter (and thus less represented) main phase of moderate storms. As $D_{s t}$ decreases from the range $[-40,-10]$ to this range $[-80,-40] \mathrm{nT}$, lifetimes drop again $\sim$ $30 \%$. Oblique waves are now strongly suppressed at latitudes above $20^{\circ}$, which partially compensates the steep wave power hike. This partial suppression of oblique waves is certainly due to higher Landau damping of oblique waves by suprathermals penetrating inward down to $L=5$ in greater numbers during geomagnetically active periods [Chen et al., 2013a]. Simultaneously, $\left\langle D_{E E}\right\rangle_{B} / E^{2}$ raises sharply with wave power, leading to an increase of $\tau_{L}\left\langle D_{E E}\right\rangle_{B} / E^{2}$ by nearly one order of magnitude. Consequently, a much stronger energization is expected to occur during such geomagnetically active periods corresponding mainly to the early recovery or main phase of moderate storms, than during less disturbed periods or quiet times. A characteristic acceleration timescale from (5) is a fraction of $\tau_{L} /\left(2 \sqrt{\tau_{L}\left\langle D_{E E}\right\rangle_{B} / E^{2}}\right)$, yielding of the order of 10 to 1 day at 1 $\mathrm{MeV}$ for $D_{s t} \sim-50$ to $-100 \mathrm{nT}$, consistent with observations [Horne et al., 2005; Fennell et al., 2012]. Corresponding lifetimes vary between about 10 and 1 day, which are also realistic values [Shprits et al., 2008a]. Lifetime and energization rate variations as a function of geomagnetic activity near $L=5$ in the outer belt are hence shown to result from a complex and nontrivial interplay between wave power increase and wave obliquity reduction over some specific latitudinal ranges as $D_{s t}$ decreases.

[17] We do not have enough statistics to study in details the lower range $D_{s t}<-100 \mathrm{nT}$ corresponding to the main phase of intense magnetic storms. Nevertheless, we can slightly extrapolate the increase of the wave intensity observed in the previous ranges. It seems that the related increase of the wave power would reduce $1 \mathrm{MeV}$ electron lifetimes to much less than 1 day, i.e., less than the main phase duration. Important precipitations of energetic electrons could therefore be expected. Moreover, additional loss processes are likely present during the main phase of a storm, such as precipitation by electromagnetic ion cyclotron waves and outward radial diffusion linked to magnetopause losses [Shprits et al., 2008a, 2008b], which may then prevent any significant and lasting acceleration from occurring. The numerical resolution of the full diffusion equation with measured initial electron distributions is left as the topic of a further work.

\section{Discussion and Conclusions}

[18] It follows from equations (1)-(3) that for a typical trough density profile $N_{e} \propto 1 / L^{4}$ and a fixed ratio $\omega_{m} / \Omega_{c e 0}$, the essential term $\tau_{L}\left\langle D_{E E}\right\rangle_{B} / E^{2} \propto\left(\Omega_{c e 0} / \Omega_{p e}\right)^{2}(\gamma+1) /(\gamma-1)$ for $D_{s t}>-40 \mathrm{nT}$ and $\propto\left(\Omega_{c e 0} / \Omega_{p e}\right)^{3 / 2}(\gamma+1)^{23 / 18} /(\gamma-1)^{13 / 18}$ for $D_{s t}<-40 \mathrm{nT}$ should vary with $L$ like $1 / L^{2}$ to $1 / L^{3 / 2}$ from low to high geomagnetic activity and for nearly constant wave obliquity. However, the obliquity of lower-band chorus waves has been observed by Cluster to shrink rapidly above $L=5.5$, even during quiet times [Agapitov et al., 2013], leading to a large increase of lifetime $\tau_{L}$ [Artemyev et al., 2013]. Accordingly, an important transition can be expected to occur around $L \sim 5.5$ from a regime of losslimited electron energization (with $\tau_{L}\left\langle D_{E E}\right\rangle_{B} / E^{2} \ll 1$ ) at lower $L$ to a regime of stronger energization independent of losses at higher $L$ (where $\tau_{L}\left\langle D_{E E}\right\rangle_{B} / E^{2} \approx 1$ as in the work by Horne et al. [2005]). This could partly account (together with magnetopause shadowing and radial diffusion) for the energetic electron flux maximum generally observed in the outer radiation belt near $L=5.5$ [Chen et al., 2007].

[19] In summary, we have investigated in this paper how electron scattering and energization vary with geomagnetic activity. It has been shown that lower-band chorus wave obliquity observed onboard Cluster abruptly decreases as $D_{s t}$ falls below $-40 \mathrm{nT}$ and that such an effect should concur with the concomitant sharp increase of wave intensity in producing a strongly enhanced energization of electrons during such active periods. Conversely, the moderate geomagnetic activity range $\left(D_{s t} \sim-40\right.$ to $\left.-10 \mathrm{nT}\right)$ should be mainly characterized by stronger losses than during quiet times, due to the relatively high oblique wave power still present. In the loss-limited regime of electron energization pertaining to the outer radiation belt, the early-time effective energization level of electrons depends critically on $\tau_{L}\left\langle D_{E E}\right\rangle_{B} / E^{2} \propto 1 / L^{2}$ instead of $\left\langle D_{E E}\right\rangle_{B} / E^{2} \propto B_{w}^{2}(L)$ in the opposite regime of negligible losses, while still increasing at lower plasma density. The variation of lower-band chorus wave obliquity with both latitude and geomagnetic activity evidenced by Cluster turns out to be an important and hitherto rather neglected parameter which needs to be included in realistic Fokker-Planck calculations of trapped electron dynamics and energization.

[20] Acknowledgments. This work was partly supported by CNES through the grant "Modele d'ondes". We thank the ESA Cluster Active Archive for providing the STAFF-SA data set. The work of A.A.V., K.V., and Z.L.M. was partially supported by The Ministry of Education and Science of Russian Federation, project 8527.

[21] The editor thanks Yuri Shprits and an anonymous reviewer for assistance evaluating this paper.

\section{References}

Agapitov, O., V. Krasnoselskikh, Y. V. Khotyaintsev, and G. Rolland (2012), Correction to "A statistical study of the propagation characteristics of whistler waves observed by Cluster", Geophys. Res. Lett., 39, L24102, doi:10.1029/2012GL054320.

Agapitov, O., et al. (2013), Statistics of whistler mode waves in the outer radiation belt: Cluster STAFF-SA measurements, J. Geophys. Res. Space Physics, 118, 3407-3420, doi:10.1029/2012JA01111.

Albert, J. M. (2009), The coupling of quasi-linear pitch angle and energy diffusion, J. Atmos. Sol. Terr. Phys., 71, 1664-1668.

Albert, J. M., and Y. Y. Shprits (2009), Estimates of lifetimes against pitch angle diffusion, J. Atmos. Sol. Terr. Phys., 71, 1647-1652, doi:10.1016/j.jastp.2008.07.004.

Artemyev, A., et al. (2012), Electron pitch-angle diffusion in radiation belts: The effects of whistler wave oblique propagation, Geophys. Res. Lett., 39, L08105, doi:10.1029/2012GL051393.

Artemyev, A., D. Mourenas, O. Agapitov, and V. Krasnoselskikh (2013), Parametric validations of analytical lifetime estimates for radiation belt electron diffusion by whistler waves, Ann. Geophys., 31, 599-624, doi:10.5194/angeo-31-599-2013.

Baker, D. N., et al. (2013), A long-lived relativistic electron storage ring embedded in EarthŠs outer Van Allen Belt, Science, 340(6129), 186-190, doi:10.1126/science. 1233518 .

Balikhin, M. A., et al. (2012), Time scaling of the electron flux increase at GEO: The local energy diffusion model vs observations, J. Geophys. Res., 117, A10208, doi:10.1029/2012JA018114. 


\section{ARTEMYEV ET AL.: DST DEPENDENCE OF LIFETIME/ENERGIZATION}

Bunch, N. L., et al. (2013), The spectral extent of chorus in the off-equatorial magnetosphere, J. Geophys. Res. Space Physics, 118, 1700-1705, doi:10.1029/2012JA018182.

Chen, Y., G. D. Reeves, and R. H. W. Friedel (2007), The energization of relativistic electrons in the outer Van Allen radiation belt, Nat. Phys., 3, 614-617, doi:10.1038/nphys655.

Chen, L., R. M. Thorne, W. Li, and J. Bortnik (2013a), Modeling the wave normal distribution of chorus waves, J. Geophys. Res. Space Physics, 118, 1074-1088, doi:10.1029/2012JA018343.

Chen, L., R. M. Thorne, Y. Shprits, and B. Ni (2013b), An improved dispersion relation for parallel propagating electromagnetic waves in warm plasmas: Application to electron scattering, J. Geophys. Res. Space Physics, 118, 2185-2195, doi:10.1002/jgra.50260.

Denton, R. E., et al. (2006), Distribution of density along magnetospheric field lines, J. Geophys. Res, 111, A04213, doi:10.1029/2005JA011414.

Fennell, J. F., S. Kanekal, and J. L. Roeder (2012), Storm responses of radiation belts during solar cycle 23: HEO satellite observations, in Dynamics of the Earth's Radiation Belts and Inner Magnetosphere, edited by D. Summers et al., 371-384, Geophys. Monogr. Ser., vol. 199, AGU, Washington, D. C. doi:10.1029/2012GM001356.

Horne, R. B., et al. (2005), Timescale for radiation belt electron acceleration by whistler mode chorus waves, J. Geophys. Res., 110, A03225, doi:10.1029/2004JA010811.

Horne, R. B., et al. (2013), Space weather impacts on satellites and forecasting the Earth's electron radiation belts with SPACECAST, Space Weather, 11, 1-18, doi:10.1002/swe.20023.

Li, W., J. Bortnik, R. M. Thorne, and V. Angelopoulos (2011), Global distribution of wave amplitudes and wave normal angles of chorus waves using THEMIS wave observations, J. Geophys. Res., 116, A12205, doi:10.1029/2011JA017035

Meredith, N. P., et al. (2012), Global model of lower band and upper band chorus from multiple satellite observations, J. Geophys. Res., 117, A10225, doi:10.1029/2012JA017978.
Mourenas, D., A. V. Artemyev, J.-F. Ripoll, O. V. Agapitov, and V. V. Krasnoselskikh (2012a), Timescales for electron quasi-linear diffusion by parallel and oblique lower-band chorus waves, J. Geophys. Res., 117, A06234, doi:10.1029/2012JA017717.

Mourenas, D., A. Artemyev, O. Agapitov, and V. Krasnoselskikh (2012b), Acceleration of radiation belts electrons by oblique chorus waves, $J$. Geophys. Res., 117, A10212, doi:10.1029/2012JA018041.

Ni, B., R. M. Thorne, N. P. Meredith, R. B. Horne, and Y. Shprits (2011), Resonant scattering of plasma sheet electrons leading to diffuse auroral precipitation: 2. Evaluation for whistler mode chorus waves, J. Geophys. Res., 116, A04219, doi:10.1029/2010JA016233.

O'Brien, T. P., and M. B. Moldwin (2003), Empirical plasmapause models from magnetic indices, Geophys. Res. Lett., 30 (4), 1152, doi:10.1029/2002GL016007.

Sheeley, B. W., M. B. Moldwin, H. K. Rassoul, and R. R. Anderson (2001), An empirical plasmasphere and trough density model: CRRES observations, J. Geophys. Res., 106, 25,631-25,642, doi:10.1029/ 2000JA000286.

Shprits, Y. Y., N. P. Meredith, and R. M. Thorne (2007), Parameterization of radiation belt electron loss timescales due to interactions with chorus waves, Geophys. Res. Lett., 34, L11110, doi:10.1029/2006GL029050.

Shprits, Y. Y., S. R. Elkington, N. P. Meredith, and D. A. Subbotin (2008a), Review of modeling of losses and sources of relativistic electrons in the outer radiation belt I: Radial transport, $J$. Atmos. Sol. Terr. Phys., 70, 1679-1693, doi:10.1016/j.jastp.2008. 06.008 .

Shprits, Y. Y., D. A. Subbotin, N. P. Meredith, and S. R. Elkington (2008b), Review of modeling of losses and sources of relativistic electrons in the outer radiation belt II: Local acceleration and loss, J. Atmos. Sol. Terr. Phys., 70, 1694-1713, doi:10.1016/j.jastp.2008.06.014.

Turner, D. L., et al. (2013), On the storm-time evolution of relativistic electron phase space density in Earth's outer radiation belt, J. Geophys. Res. Space Physics, 118, 2196-2212, doi:10.1002/jgra.50151. 